\title{
Cuidado que el medicamento no se te salga
} por la nariz

\author{
Daniela Feijoo Calles ${ }^{1}$, Lucrecia Moreno Royo², Luis Salar Ibáñez ${ }^{3}$ \\ 1. Estudiante de $5^{\circ}$ curso de Farmacia. Universidad CEU Cardenal Herrera. 2. Catedrática de Farmacología. Universidad CEU Cardenal Herrera. \\ 3. Farmacéutico comunitario. Profesor asociado. Universidad CEU Cardenal Herrera.
}

\section{PALABRAS CLAVE}

Pacientes, enfermedad pulmonar obstructiva crónica, EPOC, alumnos, aprendizaje basado en problemas, farmacéutico comunitario

\section{ABREVIATURAS}

AEMPS: Agencia Española de Medicamentos y Productos Sanitarios

BB: betabloqueantes

BDLD: broncodilatador de larga duración COPD: chronic obstructive pulmonary disease EPOC: enfermedad pulmonar obstructiva crónica

FVC: capacidad vital forzada

FEV1: volumen máximo de aire espirado en el primer segundo

GOLD: Global initiative for chronic Obstructive Lung Disease

HBP: hiperplasia benigna de próstata

HTA: hipertensión arterial

IMC: índice de masa corporal

LABA: long-acting $\beta$-agonist

LAMA: long-acting muscarinic antagonist

OMS: Organización Mundial de la Salud

PBL: aprendizaje basado en problemas

(Problem-based learning)

PRM: problema relacionado con el

medicamento

RNM: resultados negativos de la medicación

SABA: short-acting $\beta$-agonist

SAMA: short-acting muscarinic antagonist

\section{KEYWORDS}

Patients, chronic obstructive pulmonary disease, COPD, students, problem-based learning, community pharmacist

Recibido: 12/10/2017

Aceptado: 28/11/2017

Disponible online: 30-12-2017

\section{RESUMEN}

La enfermedad pulmonar obstructiva crónica (EPOC) es una patología caracterizada por una reducción persistente del flujo de aire. Esta enfermedad pulmonar es progresiva y potencialmente mortal. Suele causar disnea y las exacerbaciones y comorbilidades asociadas hacen que el pronóstico sea peor.

Se presenta un caso de seguimiento farmacoterapéutico realizado siguiendo la metodología del Foro de Atención Farmacéutica en Farmacia Comunitaria en el Aula de Práctica Farmacéutica "Paco Martínez" de la Universidad CEU Cardenal Herrera a un paciente real con EPOC y polimedicado, utilizando como método el "aprendizaje basado en problemas". Como apoyo para la evaluación de la situación se realiza un análisis de las diferentes teorías publicadas sobre la ineficacia del tratamiento. Además, se expone el nuevo enfoque que se le quiere dar al tratamiento de los pacientes con EPOC, valorando la heterogeneidad de la enfermedad y la variabilidad clínica de cada paciente mediante la revisión bibliográfica en bases de datos científicas Pubmed/Medline y Google Académico, junto con la búsqueda de información en las diferentes páginas web de organismos oficiales, Organización Mundial de la Salud y Agencia Española de Medicamentos y Productos Sanitarios.

\section{Ensure the medication is not expelled through the nose}

\section{ABSTRACT}

Chronic obstructive pulmonary disease (COPD) is a pathology characterized by a persistent reduction in airflow. This lung disease is progressive and potentially life-threatening. It can cause shortness of breath and predisposes you to severe exacerbations and illness, and symptoms gradually worsen.

A case of pharmacotherapeutic follow-up carried out in the Pharmaceutical Practice Classroom "Paco Martínez" of the Cardenal Herrera CEU University is presented to a real patient with COPD and polymedicated. It is used as a learning method based on problems. To support the evaluation of the situation, an analysis will be made of the different theories published on the ineffectiveness of treatment. In addition, the new approach to the treatment of COPD patients will be presented, assessing the heterogeneity of the disease and the clinical variability of each patient through bibliographic review in scientific databases PubMed/Medline and Google Scholar, in addition to searching for information on the different websites of official institutions, the World Health Organization and the Spanish Agency for Medicines and Health Products.

\section{Financiación: ninguna.}

Conflicto de intereses: ninguno.

Contribución a la autoría: DFC: realizó la revisión bibliográfica y colaboró en la redacción y revisión del manuscrito. LMR: redacción y revisión del manuscrito. LSI: fue el responsable del seguimiento del paciente, la exposición del caso, la redacción y revisión del manuscrito.

Cite este artículo como: Feijoo D, Moreno L, Salar L. Cuidado que el medicamento no se te salga por la nariz. Farmacéuticos Comunitarios. 2017 Dec 30; 9(4):18-25. doi:10.5672/FC.2173-9218.(2017) Vol9).004.04

Correspondencia: Luis Salar Ibáñez. (I.salar.000@micof.es)

ISSN 1885-8619 @SEFAC (Sociedad Española de Farmacia Familiar y Comunitaria). Todos los derechos reservados. 


\section{Introducción}

La enfermedad pulmonar obstructiva crónica (EPOC) es una patología caracterizada por una reducción persistente del flujo de aire. Esta enfermedad pulmonar es progresiva y potencialmente mortal y suele causar disnea (al principio asociada al esfuerzo). Las exacerbaciones y comorbilidades asociadas apuntan a un peor pronóstico. La disnea, que es persistente, al principio se asocia al esfuerzo y aumenta con el tiempo hasta aparecer en reposo [1].

Se trata de una enfermedad que no siempre se llega a diagnosticar, y puede ser mortal [1]. Según el estudio EPI-SCAN la prevalencia de EPOC en España, definida por el criterio GOLD como un cociente FEV1/FVC $<0,70$ postbroncodilatador, fue del 10,2\% y se considera que hay un infradiagnóstico del 73\% [2]. A menudo, también se utilizan los términos "bronquitis crónica" y "enfisema" para referirse a ella. La principal causa de la EPOC es la exposición al humo del tabaco (fumadores activos y pasivos), y otras causas menos frecuentes pueden ser la exposición al aire contaminado, tanto de interiores como de exteriores, así como al polvo y el humo en el lugar de trabajo [1].

Se estima que en 2015 murieron por esta causa cerca de 3 millones de personas en todo el mundo, lo cual representa un 5\% de todas las muertes registradas ese año [1]. Cada año mueren en España más de 18.000 personas a causa de la EPOC. Esta patología constituye la quinta causa de muerte entre los varones, con una tasa anual de 60 muertes por 100.000 habitantes, y la séptima para las mujeres, con una tasa anual de 17 muertes por 100.000 habitantes [3,4]. En España aproximadamente un tercio de los españoles fuma por lo que un 20-25\% desarrollarán esta enfermedad pulmonar [3,4].

Estos datos sitúan a España en un rango intermedio dentro de la Unión Europea, encabezada por algunos países de Europa del Este y anglosajones. En años venideros la Organización Mundial de la Salud (OMS) prevé un aumento dramático de estas cifras a causa de la mayor prevalencia de tabaquismo y al envejecimiento de la población en muchos países, por lo que estima que, en el año 2030, el 7,8\% de todas las muertes en el mundo serán producidas por la EPOC [1-3].

La enfermedad tiene una evolución lenta y generalmente se hace evidente a partir de los 40 o los 50 años de edad. Sus síntomas más frecuentes son la disnea (dificultad para respirar), la tos crónica y la expectoración (con mucosidad). A medida que la enfermedad empeora, los pequeños esfuerzos como subir unos cuantos escalones o llevar una maleta, o incluso las actividades cotidianas, pueden hacerse muy difíciles [1,4]. Los pacientes conforme evoluciona la enfermedad, cuando tienen una EPOC moderada o grave, suelen sufrir frecuentes exacerbaciones, es decir, episodios más intensos de disnea, tos y expectoración, que pueden durar de días a semanas. Estos episodios pueden ser muy incapacitantes, requieren atención médica de urgencia (incluso hospitalización) y, en ocasiones, pueden ser mortales.

La presencia de EPOC se sospecha en las personas que padecen los síntomas descritos anteriormente y se diagnostica mediante una prueba denominada espirometría, que mide el volumen de una espiración efectuada con un máximo esfuerzo y la rapidez con que se espira el aire [1].

La EPOC no tiene cura. Sin embargo, el tratamiento farmacológico y la fisioterapia pueden aliviar los síntomas, reducir las exacerbaciones y el intervalo entre ellas, mejorar la capacidad de ejercicio y la calidad de vida y reducir el riesgo muerte [36]. No obstante, en más de un 45\% de los casos diagnosticados de EPOC se produce una inefectividad del tratamiento farmacológico, y como consecuencia el aumento del deterioro de la calidad de vida de estos pacientes, el aumento del riesgo de muerte, y el aumento del coste sanitario [3-6].

\section{Objetivo}

En este trabajo se presenta un caso de seguimiento farmacoterapéutico de un paciente con EPOC como método del aprendizaje basado en problemas (problem-based learning) (PBL) y se realiza un análisis de las diferentes teorías publicadas sobre la ineficacia del tra- tamiento. Además, con fines didácticos, se expone el nuevo enfoque que se le quiere dar al tratamiento de los pacientes con EPOC valorando la heterogeneidad de la enfermedad y la variabilidad clínica de cada paciente.

\section{Material y método}

El estudio se ha realizado en dos partes: la primera consiste en un seguimiento farmacoterapéutico realizado en el Aula de Práctica Farmacéutica "Paco Martínez" de la Universidad CEU Cardenal Herrera de un paciente real con EPOC y polimedicado.

Y una segunda parte que consiste en la revisión bibliográfica en bases de datos científicas Pubmed/Medline y Google Académico, utilizando las palabras clave "COPD", "Obstructive disease", "epidemiology CODP data", "CODP treatment"; además de búsqueda de información en las diferentes páginas web de organismos oficiales [Organización Mundial de la Salud (OMS), Agencia Española de Medicamentos y Productos Sanitarios (AEMPS)].

\section{Procedimiento}

El primer paso es la evaluación farmacoterapéutica del paciente, para ello seguimos el diagrama de flujo del servicio de Seguimiento Farmacoterapéutico (figura 1). Se siguió la metodología Dáder [7] y los criterios de Foro de Atención Farmacéutica en Farmacia Comunitaria (figura 2) [8].

\section{Resultados y discusión Exposición del caso}

Paciente varón, de 79 años, con un sobrepeso importante $(29,4 \mathrm{~kg} /$ $\mathrm{m}^{2}$ ), exfumador con EPOC bastante avanzada a causa del consumo de tabaco durante más de 30 años. Su nivel de estudios es medio. Se trata de una persona muy inquieta y que quiere mantener una vida más activa pero su condición física se lo impide, no puede. Su mayor preocupación de salud es la EPOC porque le limita mucho en su actividad diaria. Además también padece hiperplasia benigna de próstata (HBP) e hipertensión arterial (HTA). 


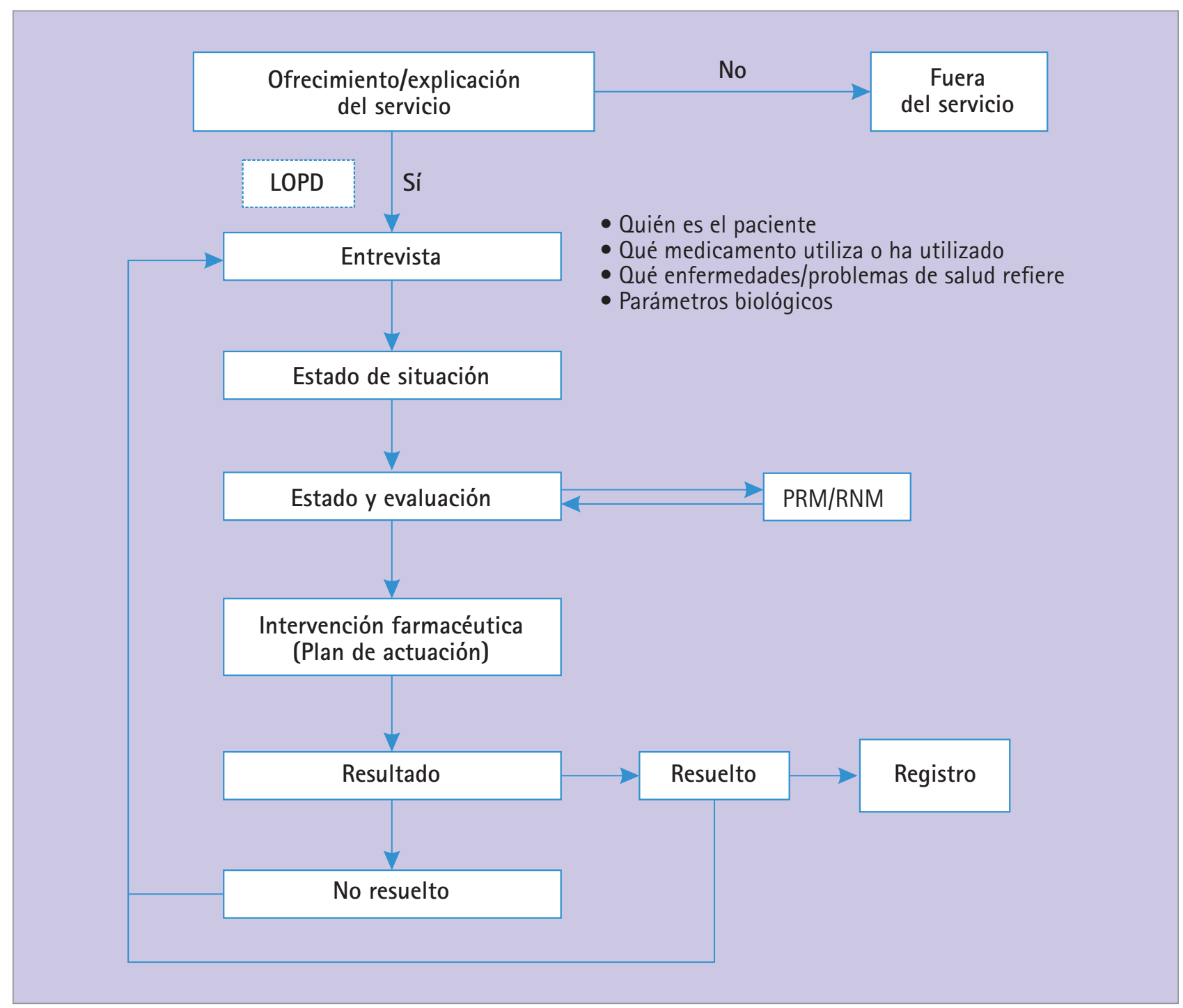

Figura 1 Diagrama de flujo del servicio de Seguimiento Farmacoterapéutico del Foro de AFen FC

Clasificación de los RNM:

\begin{tabular}{|c|c|c|}
\hline \multirow{2}{*}{ NECESIDAD } & $\begin{array}{l}\text { Necesidad de medicamento } \\
\text { (problema de salud no tratado) }\end{array}$ & $\begin{array}{l}\text { El paciente sufre un problema de salud asociado a no } \\
\text { recibir un medicamento que necesita }\end{array}$ \\
\hline & $\begin{array}{l}\text { No necesidad de medicamento } \\
\text { (efecto de medicamento innecesario) }\end{array}$ & $\begin{array}{l}\text { El paciente sufre un problema de salud asociado a recibir } \\
\text { un medicamento que no necesita }\end{array}$ \\
\hline \multirow{2}{*}{ EFECTIVIDAD } & Inefectividad no cuantitativa & $\begin{array}{l}\text { El paciente sufre un problema de salud asociado a una } \\
\text { inefectividad no cuantitativa de la medicación }\end{array}$ \\
\hline & Inefectividad cuantitativa & $\begin{array}{l}\text { El paciente sufre un problema de salud asociado a una } \\
\text { inefectividad cuantitativa de la medicación }\end{array}$ \\
\hline \multirow{2}{*}{ SEGURIDAD } & Inseguridad no cuantitativa & $\begin{array}{l}\text { El paciente sufre un problema de salud asociado a una } \\
\text { inseguridad no cuantitativa de un medicamento }\end{array}$ \\
\hline & Inseguridad cuantitativa & $\begin{array}{l}\text { El paciente sufre un problema de salud asociado a una } \\
\text { inseguridad cuantitativa de un medicamento }\end{array}$ \\
\hline
\end{tabular}

Figura 2 Clasificación de los resultados negativos de la medicación (RNM) (Foro de AFen FC) 


\begin{tabular}{|c|c|c|c|c|}
\hline Edad: 79 & Sexo: $\vdash$ & Peso: 80 kg & Talla: $165 \mathrm{~cm}$ & IMC: $29,4 \mathrm{~kg} / \mathrm{m}^{2}$ \\
\hline \multicolumn{2}{|l|}{ Patología } & \multicolumn{2}{|l|}{ Medicamento } & Posología \\
\hline \multicolumn{2}{|c|}{ 401.9 Hipertensión } & \multicolumn{2}{|c|}{$\begin{array}{l}\text { Coaprove|}{ }^{\circledR} \text { (irbesartán/ } \\
\text { hidroclorotiazida) 300/12,5 mg }\end{array}$} & $1-0-1 / 2$ \\
\hline \multirow{5}{*}{\multicolumn{2}{|c|}{ 491.2 EPOC }} & \multicolumn{2}{|c|}{ Spiriva Respimat ${ }^{\circledR}$ (tiotropio) 2,5 mcg } & 2/dia \\
\hline & & \multicolumn{2}{|c|}{$\begin{array}{l}\text { Duoresp Spiromax }{ }^{\circledR} \text { (formoterol/ } \\
\text { budesonida) }\end{array}$} & 1/dia \\
\hline & & \multicolumn{2}{|c|}{ Ventolin ${ }^{\circledR}$ (salbutamol) 100 mcg } & A demanda \\
\hline & & \multicolumn{2}{|l|}{ Prednisona } & En exacerbaciones \\
\hline & & \multicolumn{2}{|c|}{ Levofloxacino $500 \mathrm{mg}$} & En exacerbaciones \\
\hline \multicolumn{2}{|l|}{ Taquicardias } & \multicolumn{2}{|c|}{ Corlentor ${ }^{\circledR}$ (ivabradina) $5 \mathrm{mg}$} & $1 / 2-0-1 / 2$ \\
\hline \multicolumn{2}{|l|}{ Dolor } & \multicolumn{2}{|c|}{ Coslan ${ }^{\circledR}$ (ácido mefenámico) } & A demanda \\
\hline \multicolumn{2}{|c|}{ 536.8 Dispepsia } & \multicolumn{2}{|c|}{ Opiren ${ }^{\circledR}$ (lansoprazol) $30 \mathrm{mg}$} & $1-0-0$ \\
\hline \multicolumn{2}{|c|}{$\begin{array}{l}\text { Hiperplasia benigna } \\
\text { de próstata }\end{array}$} & \multicolumn{2}{|c|}{ Urorec $^{\circledR}$ (silodosina) 8 mg } & $1-0-0$ \\
\hline \multicolumn{2}{|c|}{780.52 Insomnio } & \multicolumn{2}{|c|}{$\begin{array}{l}\text { Lorazepam } 1 \text { mg (a demanda, una } \\
\text { semana) }\end{array}$} & $0-0-1$ \\
\hline
\end{tabular}

Tabla 1 Medicación que tiene prescrita el paciente

La medicación que le ha sido prescrita por los diferentes especialistas se refleja en la tabla 1.

Durante la entrevista el paciente nos indica que se controla la saturación de oxígeno frecuentemente, porque algunas veces olvida aplicarse el broncodilatador (tiotropio 2,5 mcg) $\mathrm{y}$ esto le hace sentirse peor, incluso ha tenido que ir a urgencias en dos ocasiones para que le estabilizaran. También dice que toma éste conjuntamente con Duoresp ${ }^{\circledR}$ (formoterol/budesonida) 16/4,5 mcg por la mañana, ambos a la vez y en ayunas. Que utiliza salbutamol 100 mcg, unas 4 o 5 veces al día y que, en ocasiones, cuando tiene exacerbaciones de la EPOC, utiliza prednisona durante unos días.

Nos dice que toma ivabradina 5 mg porque empezó a tener taquicardias y que le ha regulado bien la frecuencia cardíaca, pasando de 98 a 70 pulsaciones por minuto.

Para la HTA toma irbersartan con hidroclorotiazida $(300 / 12,5 \mathrm{mg})$, uno por la mañana y medio antes de acostarse. Y para la HBP se le ha recetado silodosina $8 \mathrm{mg}$. Refiere que esto le ha ayudado a tener que levantarse menos veces a orinar por la noche, que era lo que más le incomodaba, pasando de 4 a 2 veces.

Toma lansoprazol $30 \mathrm{mg}$ todos los días como protector gástrico. Y refie- re dificultad para poder dormir, por lo que se le ha prescrito lorazepam $1 \mathrm{mg}$, pero que toma esporádicamente.

También nos indica que en ocasiones toma Coslán ${ }^{\circledR}$ (ácido mefenámico) $250 \mathrm{mg}$, un analgésico poco común, para el dolor de cabeza, porque trabajó de visitador médico en el laboratorio fabricante de este medicamento, y a él le funciona muy bien.

Por último, nos comenta que tiene un sobrino médico que le receta el antibiótico levofloxacino $500 \mathrm{mg}$. Debe tenerlo en casa y tomarlo durante siete días cuándo sienta que está en un proceso catarral, e incluso antes de que comiencen los síntomas en temporada de catarros para prevenir complicaciones.

Añade que tiene sequedad de boca y en la conversación comenta que se encuentra muy excitado y eso le hace discutir con su mujer con más frecuencia de lo que le gustaría.

\section{Estudio y evaluación del tratamiento}

La EPOC es una enfermedad muy heterogénea, y durante muchos años no se han tenido en cuenta las peculiaridades (los diferentes fenotipos) de los pacientes a la hora de recomendar su tratamiento [4]. En gran parte esto se ha debido a que las opciones

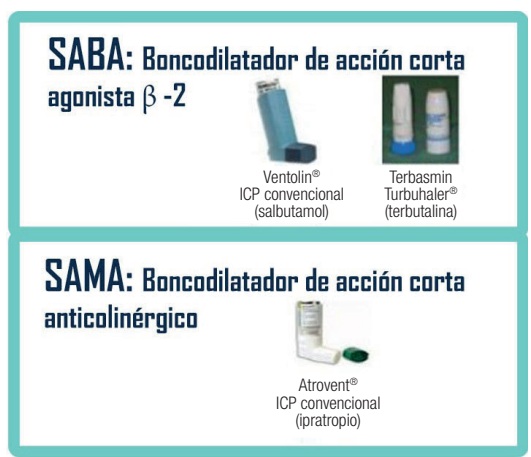

Figura 3 Clasificación de los

broncodilatadores de rescate SABA y SAMA

terapéuticas eran escasas y no existía evidencia de que su eficacia fuera significativamente diferente en diversos tipos de pacientes. El gran desarrollo de diferentes opciones en el tratamiento farmacológico y no farmacológico ha comportado la demostración de que la respuesta clínica puede ser diferente según las características de la enfermedad.

Por regla general el primer paso en el tratamiento de la EPOC es la broncodilatación. Los broncodilatadores de acción corta anticolinérgicos-SAMA (short-acting muscarinic antagonist) como el bromuro de ipratropio, y los $\beta_{2}$ agonistas de acción corta-SABA (short-acting $\beta$-agonist) como el salbutamol o la terbutalina, son los fármacos eficaces en el control rápido de los síntomas (figura 3). Estos fármacos, añadidos al tratamiento de base, son de elección para el tratamiento a demanda de los síntomas, sea cual sea el nivel de gravedad de la enfermedad.

Cuando el paciente tiene síntomas frecuentes, precisa tratamiento con broncodilatadores de corta duración de forma frecuente o cuando sufre una limitación al ejercicio requiere tratamiento de base regular. En este caso se debe administrar un broncodilatador de larga duración (BDLD): LAMA o LABA, según sean antimuscarínicos (long-acting muscarinic antagonist) o agonistas $\beta_{2}$ (long-acting $\beta$-agonist) (figura 4).

En pacientes sintomáticos o con una limitación al ejercicio evidente aun tras la monoterapia broncodilatadora, se debe ensayar la doble terapia broncodilatadora. La asociación de LABA y LAMA ofrece un beneficio funcional añadido con reducción de la necesidad de medicación de rescate y mejoría de los síntomas y de la calidad de vida frente a la monoterapia. Por este motivo, en un segundo 


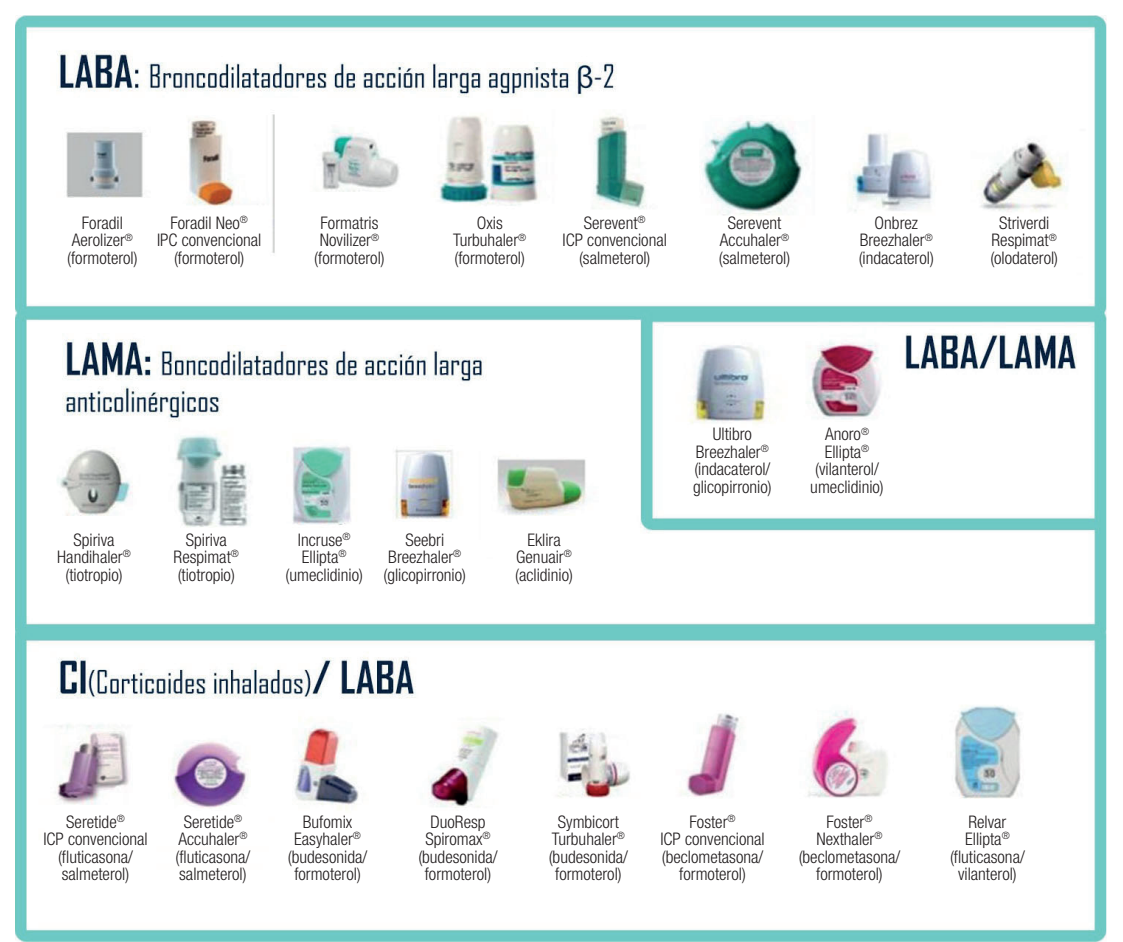

Figura 4 Clasificación de los broncodilatadores de mantenimiento

escalón de tratamiento, en pacientes con un nivel de gravedad II en adelante se puede asociar un BDLD de un tipo farmacológico distinto al que el paciente llevara en el primer nivel de gravedad, sea LAMA o LABA. De esta manera se consigue optimizar el efecto broncodilatador.

El paciente lleva pautado para su EPOC dos inhaladores: uno con tiotropio (Spiriva Respimat ${ }^{\circledR}$ LAMA) $\mathrm{y}$ otro con formoterol (LABA) y budesonida. Y además, Ventolin ${ }^{\circledR}$ (salbutamol SABA) como medicamento de rescate para uso a demanda.

Tal y cómo hemos detectado en la entrevista, el paciente utiliza a demanda Ventolin ${ }^{\circledR}$ con mucha frecuencia, un envase le dura menos de un mes. La dosis máxima en 24 horas es de 8 aplicaciones (800 $\mu \mathrm{g})$. Esta elevada dosis de salbutamol le podría estar produciendo la reacción adversa de taquicardia.

En cuanto a los efectos adversos más frecuentes de salbutamol, encontramos además de la taquicardia, temblores y dolor de cabeza. También como efecto adverso muy raro, en el prospecto aparece la hiperactividad, agitación y la consecuente sobreexcitación que percibimos al realizarle la entrevista. Estos últimos también manifestados por el paciente.

Esta situación de excesiva necesidad de broncodilatación nos alertaría de la necesidad de tratamiento con corticoides de mantenimiento, si no lo llevara ya pautado.

Por lo tanto, la siguiente medida a tomar es confirmar que utiliza bien el inhalador. Tras chequear la técnica, su farmacéutico detecta que no usa correctamente el inhalador Spiriva Respimat ${ }^{\circledR}$. No hace la apnea posterior a la inhalación y se aprecia que le sale una nubecilla por la nariz. El medicamento se le sale por la nariz por lo que tiene una infradosificación del inhalador LAMA.

En la HTA los betabloqueantes (BB) son los fármacos más eficaces para reducir la frecuencia cardiaca. En los pacientes con enfermedad coronaria, a menos que existan contraindicaciones específicas, los BB representan fármacos de primera línea. Las contraindicaciones para el uso de BB incluyen la EPOC y el asma, entre otras. En estos enfermos, los BB pueden motivar un aumento de la reactividad de la vía aérea y espasmo bronquial y reducir la respuesta a los agonistas beta adrenérgicos, administrados por vía oral o inhalatoria.

Ivabradina es un fármaco que forma parte de los agentes selectivos y específicos para reducir la frecuencia cardiaca actuando en el nódulo sinusal mediante la inhibición de la corriente If y sin modificar la contractilidad del corazón, la repolarización o la conducción auriculoventricular. Sus indicaciones autorizadas inclu- yen el tratamiento de insuficiencia cardiaca y los sintomas de angina de pecho estable crónica en determinados pacientes y condiciones clínicas. Por sus propiedades, en particular por la falta de interacción con los receptores beta adrenérgicos, ivabradina puede ser especialmente útil en los pacientes con enfermedades bronquiales obstructivas.

Silodosina es un bloqueante alfa adrenérgico. Alivia los síntomas de la HBP al relajar los músculos de la vejiga urinaria y la próstata.

Levofloxacino es un antibiótico del grupo de las quinolonas, más concretamente una fluoroquinolona. Se trata de un enantiómero activo de ofloxacino con casi el doble de la potencia y con una sustancial menor toxicidad. Al igual que otras quinolonas, actúa al inhibir el enzima ADN girasa, encargado del empaquetamiento del ADN. Es efectivo frente a un buen número de bacterias Gram positivas y Gram negativas, por lo que se considera un antibiótico de amplio espectro. Por esa razón, se acostumbra a administrar levofloxacino de manera empírica en infecciones como la neumonía bacteriana adquirida en la comunidad o infecciones urinarias, antes de que se conozca el organismo causal específico. Una vez identificado el germen etiológico, se puede omitir levofloxacino a cambio de un antibiótico de espectro más específico, por lo que no está recomendado como antibiótico de primera línea, para evitar resistencias.

Este medicamento tiene efectos secundarios muy similares a los de otras quinolonas. Algunas de las reacciones adversas más severas asociadas con la administración de este grupo de medicamentos incluyen daño neuronal permanente, aumento en la prolongación del segmento QT cardíaco, y algunos eventos del sistema nervioso central como agitación e insomnio crónico. Todo esto, con el historial del paciente, debería ser suficiente para que al menos se valorara su necesidad y seguridad en los casos en los que muchas veces no existe una infección compleja.

Haciendo un análisis exhaustivo de la situación farmacoterapéutica del paciente observamos que:

- El tratamiento de base de la EPOC no está bien ajustado porque tiene la necesidad de consumir en exceso el salbutamol. Esto puede ser causa 
de taquicardias, una reacción adversa frecuente del broncodilatador.

- Ivabradina, pese a no ser el tratamiento de elección para las taquicardias, está indicada en este paciente.

- La sequedad de boca puede estar producida por no realizar los enjuagues suficientes entre la administración de Spiriva ${ }^{\circledR}$ y Duoresp ${ }^{\circledR}$.

- Hidroclorotiazida, al ser adminis- trada por la noche le puede hacer ir más al baño.

- Lansoprazol solamente lo debe tomar cuando tome prednisona.

- Levofloxacino es un antibiótico de reserva y solamente debería ser utilizado en algunos casos para evitar las resistencias bacterianas.

- Y, finalmente, el sobrepeso exacerba los síntomas y signos de la EPOC, simplemente por una cues- tión mecánica, de espacio para expandir los pulmones.

Centrándonos en esto último se pretende observar si la ineficacia en el tratamiento pudiera estar causada por la variabilidad entre los distintos inhaladores que hay en el mercado. Longest y cols., en un estudio en el que compararon varios tipos de inhaladores [9], observaron (figura 5) que el

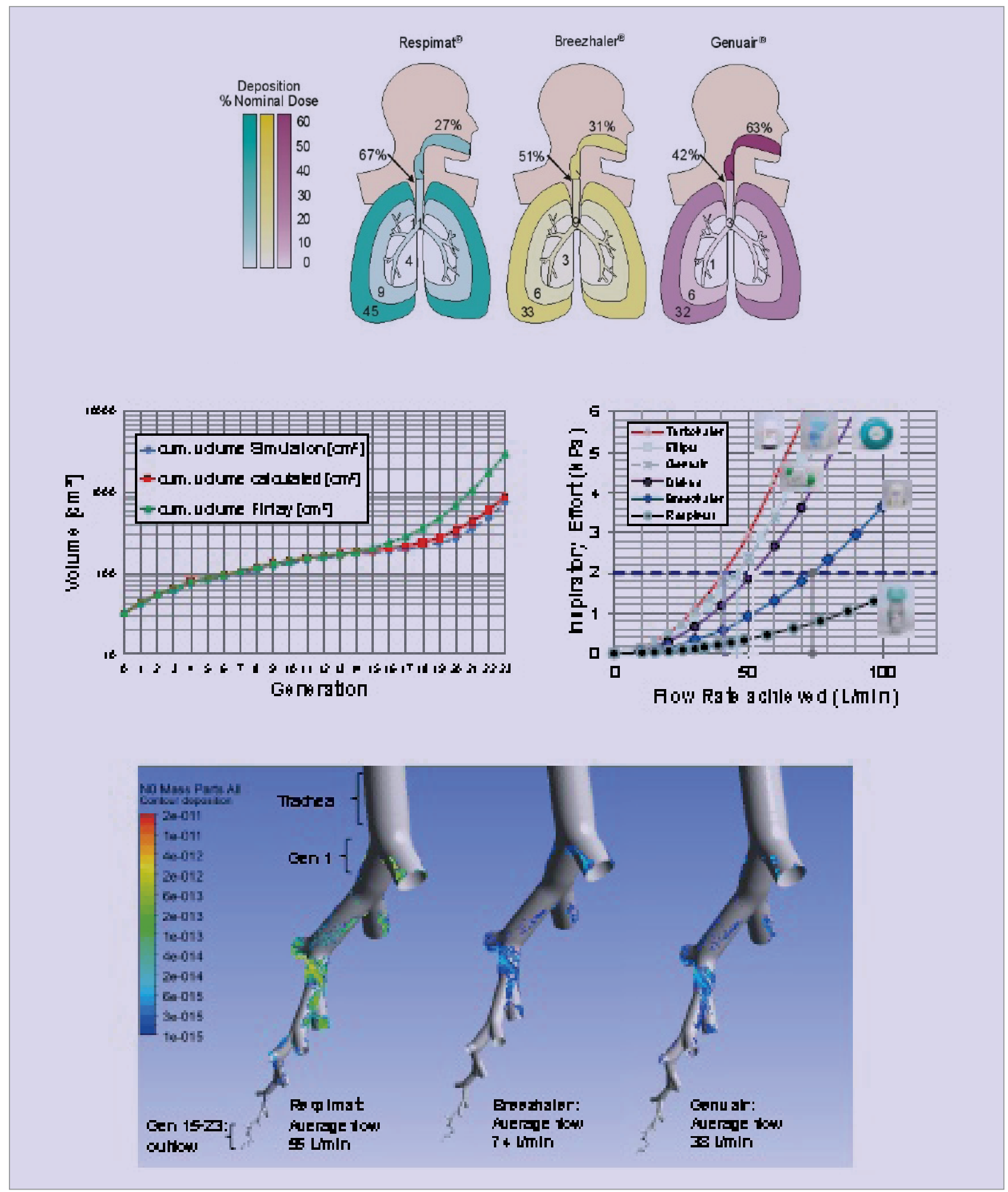

Figura 5 Estudio comparativo de varios tipos de inhalador 


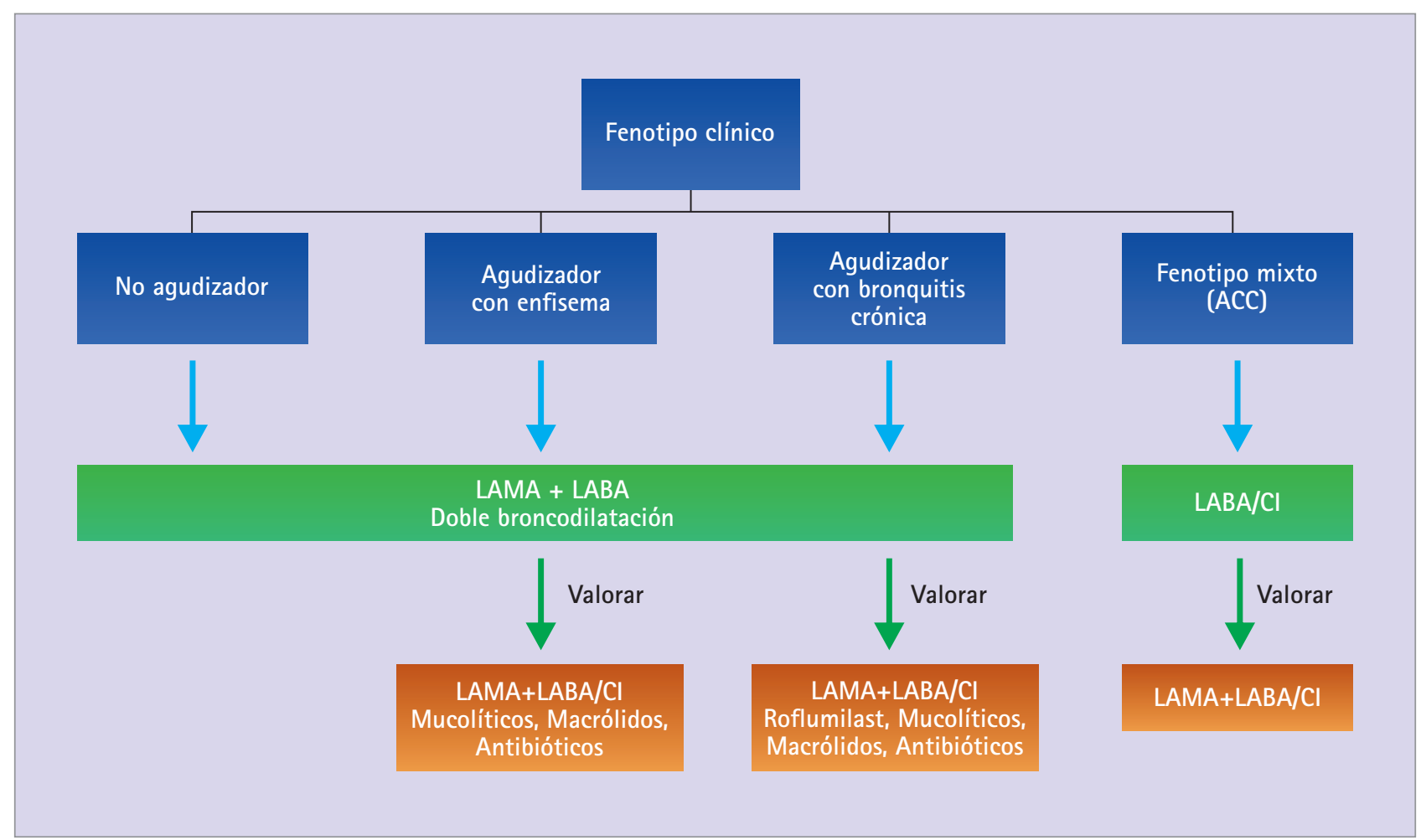

Figura 6 GesEPOC. Estrategia de abordaje de la enfermedad [6]

inhalador que mejor ejerce su acción es Respimat ${ }^{\circledR}$ pues es el que más llega al lugar de acción y, aun así, podría no ser suficiente, al llegar solamente el 45\% del fármaco, y producirse una infradosificación, y la consecuente inefectividad terapéutica [9].

Además, hasta ahora, las guías clínicas no establecen diferencias entre los diferentes niveles de afección que tienen los pacientes, o subtipos de la enfermedad, a pesar de que, la EPOC, como se ha dicho anteriormente, es una enfermedad especialmente heterogénea y de esto surge la necesidad de reestructurar la forma en que se aborda cada caso.

\section{Nuevas estrategias en el tratamiento de la EPOC}

El término fenotipo aplicado a la EPOC [7] se define como "aquellos atributos de la enfermedad que solos o combinados describen las diferencias entre individuos con EPOC en relación a parámetros que tienen significado clínico". De entre todos los descritos, existen tres que se asocian con factores pronósticos y sobre todo con distinta respuesta a los tratamientos disponibles en la actualidad. Estos fenotipos son: el agudizador, el mixto EPOC-asma y el enfisema-hiperinsuflado [10].
El agudizador se caracteriza por la presencia de al menos dos agudizaciones el año, y además del tratamiento con broncodilatadores de larga duración puede requerir la utilización de fármacos antiinflamatorios. El fenotipo mixto presenta una obstrucción no completamente reversible al flujo aéreo acompañada de una reversibilidad aumentada de la obstrucción. Por su perfil inflamatorio subyacente suele presentar una buena respuesta terapéutica a los corticosteroides inhalados unidos a los broncodilatadores. Por último, el fenotipo enfisema presenta una pobre respuesta a los fármacos antiinflamatorios de que disponemos en la actualidad, y los broncodilatadores de larga duración, junto a la rehabilitación, son la base de su tratamiento [10].

En este sentido, la simple pregunta: ¿Cuántas agudizaciones sufrió el año anterior? va a permitir la clasificación como agudizador si la respuesta es dos o más, sean cuales sean las características clínicas o funcionales del paciente, ya que el tratamiento debe dirigirse de forma prioritaria a la prevención de las agudizaciones. Si la respuesta es una o ninguna, debemos investigar si se trata de un enfisema o un fenotipo mixto. El reconocimiento de las peculiaridades de los distintos fenotipos de la EPOC nos debe permitir guiar un tratamiento más personalizado en el que las características del paciente se sumen a su gravedad para dirigir la terapia. El siguiente paso será el reconocimiento de estos fenotipos clínicos en las nuevas normativas de tratamiento de la EPOC.

Así pues, la GesEPOC sigue una estrategia de abordaje de la enfermedad que puede verse resumida en el esquema de la figura 6 [10].

Recientemente se ha publicado la versión 2017 de la estrategia Global initiative for chronic Obstructive Lung Disease (GOLD) [10]. Esta versión es un paso más de un proceso que se inició en 2001 con el primer documento, que contribuyó de forma decisiva a situar a la enfermedad pulmonar obstructiva crónica (EPOC) en el mapa de las enfermedades relevantes para la salud pública a nivel mundial y también a estandarizar aspectos como su definición, criterios diagnósticos y pautas generales de tratamiento.

\section{Intervención}

Tras la evaluación, pensamos que las prescripciones de nuestro paciente son las apropiadas para su situación, y el inhalador que utiliza es el más adecuado de los disponibles. Aun así, 


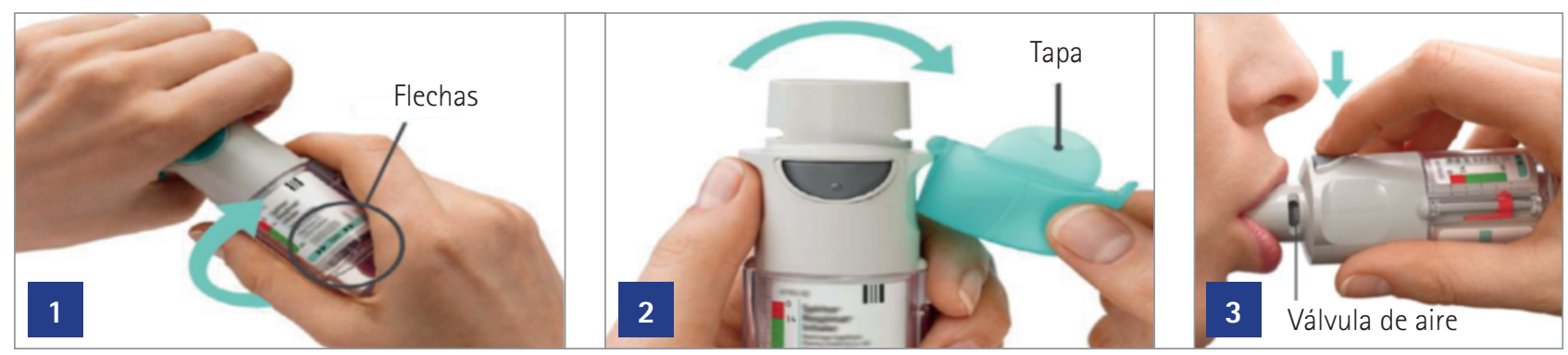

Figura 7 Pasos para la administración del inhalador de cartucho presurizado

se observan los síntomas correspondientes a la infradosificación del inhalador Spiriva Respimat ${ }^{\circledR}$ como se ha indicado anteriormente. Se procede pues a la evaluación de la técnica de administración que sigue, y tras observarlo, se comprueba que no realiza correctamente los pasos mediante los que se debe administrar el inhalador (figura 7):

1. Rotar. Mantenga la tapa cerrada. Rote la base transparente en la dirección de las flechas de la etiqueta hasta que haga clic (media vuelta)

2. Abrir. Abra la tapa completamente.

3. Presionar. Espire lenta y profundamente.

a. Cierre los labios alrededor de la boquilla sin cubrir las válvulas de aire.

b. Apunte con el inhalador hacia el fondo de la garganta.

c. Mientras inspira lenta y profundamente a través de la boca, presione el botón de liberación de dosis y continúe inspirando lentamente mientras le resulte cómodo.

d. Mantenga la respiración durante 10 segundos o hasta que le sea posible.

e. Cierre la tapa hasta que vuelva a utilizar el inhalador

4. Repita los pasos 1 al 3 para completar el total de 2 pulsaciones

Se comprueba que el paciente no realiza el paso 3-d en el que se produce la apnea necesaria para la retención y penetración del fármaco, por lo que se le dan las instrucciones necesarias, comprobando que las entiende e incorpora a su procedimiento habitual de administración del medicamento.

\section{Conclusiones}

Tras evaluar todos los datos y analizar los distintos factores que podrían afectar a la infradosificación del paciente se concluye que el problema, en este caso, no ha estado en la pauta terapéutica, ni en el fallo del tratamiento per se. Sino más bien, se observa que el PRM (problema relacionado con el medicamento) de mala utilización del dispositivo de administración ha sido producido por un fallo en la educación sanitaria que se requiere en este tipo de patologías. El paciente, a pesar de ser a primera vista una persona con formación en el ámbito sanitario, ha tenido problemas con la adherencia y la autoadministración de sus medicamentos, por lo que nos planteamos la revisión de la técnica, al menos una vez cada dos meses.

Para evitar problemas de efectividad y seguridad de la farmacoterapia cabe insistir en la necesidad de la colaboración multidisciplinar en el ámbito de la salud, para que este tipo de problemas puedan prevenirse.

Para que los pacientes logren poseer las competencias necesarias para un uso adecuado de las distintas formas farmacéuticas que garanticen la efectividad esperada en la medicación es imprescindible la implicación de todos los profesionales sanitarios situados en el entorno del medicamento. En este aspecto, la atención farmacéutica y el seguimiento farmacoterapéutico, incluyendo en sus procedimientos las acciones de educación sanitaria, juegan un papel fundamental para comprobar siempre que la necesidad, efectividad, y seguridad de los fármacos esté asegurada.

\section{Referencias bibliográficas}

1. Organización Mundial de la Salud [Internet]. Enfermedad pulmonar obs- tructiva crónica (EPOC). 2017. [Acceso 17/8/2017]. Disponible en: http:// www.who.int/mediacentre/factsheets/ fs315/en/

2. Miravitlles M, Soriano JB, Garcia-Rio F, Muñoz L, Duran-Tauleria E, Sanchez G, et al. Prevalence of COPD in Spain: impact of undiagnosed COPD on quality of life and daily life activities. Thorax 2009; 64(10):863-868.

3. Soriano JB, Miravitlles M. Datos epidemiológicos de EPOC en España. Arch Bronconeumol. 2007; 43(1):2-9.

4. Guía española de la EPOC (GesEPOC) [Internet]. Revisado 27/2/2017. [Acceso 17/8/2017]. Disponible en: http:// www.gesepoc.com/entrada_ficha. php?IDent $=114$

5. Miravitlles M. Prevention of exacerbations of COPD with pharmacotherapy. Eur Respir Rev. 2010; 19:119-126.

6. Alfageme Michavila I, Reyes Núñez N, Merino Sánchez M, Gallego Borrego J. Fármacos anticolinérgicos. Arch Bronconeumol. 2007; 43(Supl 4):3-10.

7. Sabater Hernández D, Silva Castro MM, Faus Dáder MJ. Programa Dáder de Seguimiento Farmacoterapéutico. [Monografía en Internet]. 2007. [Acceso 16/8/2017]. Disponible en: http://www.ugr.es/ cts131/esp/guias/ GUIA\%20FINAL\%20DADER.pdf

8. Foro AF-FC [Internet]. Guía Práctica para los Servicios de Atención Farmacéutica en la Farmacia Comunitaria. Consejo General de Colegios Oficiales de Farmacéuticos. 2010. [Acceso 17/8/2017]. Disponible en: http://www.portalfarma.com/inicio/atencionfarmaceutica/ forofarmaciacomunitaria/Documents/ ATFC_Guia\%20FORO.pdf

9. Longest PW, Tian G, Walenga RL, Hindle M. Comparing MDI and DPI aerosol deposition using in vitro experiments and a new stochastic individual path (SIP) model of the conducting airways. Pharm Res. 2012 Jun; 29(6):1670-1688. doi:10.1007/ s11095-012-0691-y

10. Miravitlles M, Soler JJ. GOLD en 2017: una visión desde la Guía Española de la EPOC (GesEPOC). Arch Bronconeumol 2017; 53:89-90. 